7.- De Turck B J, Diltoer M W, Cornelis P J, Maes V, Spapen H D, Camu F, et al. Lowering of plasma valproic acid concentrations during concomitant therapy with meropenem and amikacin. J Antimicrob Chemother 1998; 42: 563-4.

8.- Nacarkucuk E, Saglam H, Okan M. Meropenem decreases serum level of valproic acid. Pediatr Neurol 2004; 31: 232-4.

9.- Nakajima Y, Mizobuchi M, Nakamura M, Takagi H, Inagaki H, Kominami G, et al. Mechanism of the drug interaction between valproic acid and carbapenem antibiotics in monkeys and rats. Drug Metab Dispos 2004; 32: 1383-91.

10.- Suzuki E, Yamamura N, Ogura Y, Nakai D, Kubota K, Kobayashi N, et al. Identification of valproic acid glucuronide hydrolase as a key enzyme for the interaction of valproic acid with carbapenem antibiotics. Drug Metab Dispos 2010; 38: 1538-44.

11.- Suzuki E, Nakai D, Yamamura N, Kobayashi N, Okazaki O, Izumi T. Inhibition mechanism of carbapenem antibiotics on acylpeptide hydrolase, a key enzyme in the interaction with valproic acid. Xenobiotica 2011; 41: 958-63.
12.- Mori H, Takahashi K, Mizutani T. Interaction between valproic acid and carbapenem antibiotics. Drug Metab Rev 2007; 39: 647-57.

13.- Haroutiunian S, Ratz Y, Rabinovich B, Adam M, Hoffman A. Valproic acid plasma concentration decreases in a dose-independent manner following administration of meropenem: a retrospective study. J Clin Pharmacol 2009; 49: 1363-9.

14.- Spriet I, Goyens J, Meersseman W, Wilmer A, Willems L, Van Paesschen W. Interaction between valproate and meropenem: a retrospective study. Ann Pharmacother 2007; 41: 1130-6.

15.- Santucci M, Parmeggiani A, Riva R. Seizure worsening caused by decreased serum valproate during meropenem therapy. J Child Neurol 2005; 20: 456-7.

16.- Williams N T. Medication administration through enteral feeding tubes. Am J Health Syst Pharm 2008; 65: 2347-57.

17.- Astrazeneca. Ficha técnica Meropenem. http://www1.astrazeneca-us.com/pi/ MerremIV.pdf [accedido el 30-08-2011].

18.- Coves-Orts F J, Borrás-Blasco J, Navarro-Ruiz A, Murcia-López A, Palacios-Ortega F. Acute seizures due to a probable interaction between valproic acid and meropenem. Ann Pharmacother 2005; 39: 533-7.

\section{Bacteriemia por Kocuria rosea en un paciente con SIDA}

\author{
Marcelo Corti, María F. Villafañe, Isabel Soto, \\ Omar Palmieri y Raquel Callejo
}

\section{Bacteremia by Kocuria rosea in an AIDS patient}

Kocuria rosea is an uncommon pathogen may cause opportunistic infections in immunocompromised patient. We report a HIV patient, who presented bacteremia caused by Kocuria rosea. He was successfully treated with vancomycin and by catheter removal.

Key words: Kocuria rosea, HIV, immunocompromised patient, bacteremia.

Palabras clave: Kocuria rosea, VIH, paciente inmunocomprometido, bacteriemia.

\section{Introducción}

E 1 género Kocuria incluye 11 especies, de las cuales hasta la fecha sólo tres han sido involucradas como patógenas para el ser humano. Estas son: $K$. rosea, $K$. varians y K. kristinae, las que fueron identificadas, aunque con poca frecuencia, como causa de enfermedad en pacientes inmunocomprometidos ${ }^{1}$.

Luego de la revisión taxonómica efectuada por Stackebrandt et al. ${ }^{1}$ en 1955, el género Micrococcus se dividió en 5 subgéneros que incluyen: Micrococcus, Nesterenkonia, Kytococcus, Dermacoccus y Kocuria. De esta forma, Kocuria sp. es un miembro de la familia Micrococcaceae formada por cocáceas grampositivas aerobias, no capsuladas y no formadoras de endosporas ${ }^{2}$.

Hospital de Enfermedades Infecciosas Francisco J. Muñiz, Buenos Aires, Argentina.

División B, VIH/SIDA (MC, MFV, IS, OP). Laboratorio de Bacteriología (RC).

Recibido: 30 de junio de 2011 - Aceptado: 23 de enero de 2012

Correspondencia a:

Marcelo Corti

marcelocorti@fibertel.com.ar
En la literatura médica sólo existen escasas comunicaciones de pacientes inmunocomprometidos con infecciones asociadas con catéteres vasculares, bacteriemia y colecistitis aguda en los que se identificó a especies de Kocuria como agentes causales ${ }^{3,4}$.

Presentamos el caso de un paciente con infección por VIH que desarrolló una bacteriemia por $K$. rosea asistido en el Hospital de Referencia para Enfermedades Infecciosas F. J. Muñiz de la ciudad de Buenos Aires.

\section{Caso clínico}

Paciente varón, de 60 años, con diagnóstico reciente de infección por $\mathrm{VIH}$, sin tratamiento anti-retroviral, consumidor de tabaco (20 cigarrillos diarios), sin antecedentes de enfermedades oportunistas. Ingresa con síndrome febril, disnea clase funcional II/III, tos no productiva y pérdida de peso de tres meses de evolución. Al ingreso, en la exploración física destacaba una onicomicosis de ambos pies, dermatitis ocre en ambos miembros inferiores, xerodermia, mal estado de sus piezas dentarias, disminución de la ventilación pulmonar, con roncus y sibilancias bilaterales, taquicardia de 120 lpm y taquipnea de 26 respiraciones por minuto. En la radiografía de tórax se observaba un infiltrado intersticio-alveolar micronodulillar bilateral. El laboratorio de ingreso mostró discreta anemia, VHS acelerada $\left(97 \mathrm{~mm} 1^{\text {a }}\right.$ hora), leucocitos: $9800 / \mathrm{mm}^{3}$, función renal y hepática normal, saturación de oxígeno 89\%, LDH $1049 \mathrm{U} / \mathrm{L}$. El recuento de linfocitos T CD4 + fue < de 50 céls $/ \mathrm{mm}^{3}$. La serología para hepatitis B y C resultó no reactiva. La tinción de Ziehl-Neelsen en el examen de esputo resultó negativa así como los exámenes directos y cultivos para bacterias comunes y hongos. Los hemocultivos tampoco mostraron desarrollo de microorganismos. Se inició tratamiento antibiótico empírico con ampicilina/sulbactam 1,5 gr cada 6 horas por vía iv, cotrimoxazol $800 / 160 \mathrm{mg}$ cada 6 horas iv y dexametasona $24 \mathrm{mg}$ /día iv. Se agregó al tratamiento oxígeno humidificado al 28\%. El paciente evolucionó de manera desfavorable con deterioro de su cuadro clínico, hipoxemia con una $\mathrm{PO}_{2}$ de $64 \mathrm{mmHg}$ con acidosis metabólica y alcalosis respiratoria sin signos de falla de bomba. Se realizó fibrobroncoscopia con lavado broncoalveolar de donde se aisló Histoplasma capsulatum y con la coloración de Grocott se observaron estructuras compatibles con Pneumocystis jiroveci. Los hemocultivos también se aislaron levaduras compatibles con $H$. capsulatum. Se inició tratamiento con anfotericina B deoxicolato $0,7 \mathrm{mg} / \mathrm{kg} /$ día durante 14 días y luego se indicó itraconazol $400 \mathrm{mg} /$ día y se mantuvo el tratamiento para $P$. jiroveci hasta completar 21 días de tratamiento. El paciente mejoró su estado clínico y a los 30 días de internación 
presenta nuevo episodio febril con signos clínicos de bacteriemia (fiebre, escalofríos, taquicardia e hipotensión arterial). Se realizaron dos nuevos hemocultivos de sangre periférica con el sistema BactAlert (bioMerieux $®$ ) y las muestras fueron subcultivadas en agar sangre de cordero y agar chocolate e incubadas a $35^{\circ} \mathrm{C}$ durante 48 horas en una atmósfera de $5 \%$ de $\mathrm{CO}_{2}$. El examen microscópico directo mostró cocáceas grampositivas en racimos; ambos cultivos desarrollaron colonias circulares, ligeramente convexas, con pigmento rosado, no hemolíticas, catalasa positiva y coagulasa negativa. La identificación preliminar se realizó de acuerdo con un esquema bioquímico para la diferenciación de géneros dentro de las cocáceas grampositivas catalasa positiva. Se utilizaron las siguientes pruebas fenotípicas: desarrollo en $\mathrm{NaCl}$ al 6,5\%, prueba de bencidina positiva, producción de ácido de glucosa en anaerobiosis, sensibilidad al disco de bacitracina $0.04 \mathrm{U}$ y a lisozima y resistencia a furazolidona de $100 \mu \mathrm{g} /$ disco y a lisostafina. El sistema Vitek 2 (bioMerieux ${ }^{\circledR}$ ) permitió identificar el aislado como $K$. rosea con una probabilidad de $96 \%$. El gen $16 \mathrm{~S}$ ARNr se amplificó utilizando los partidores universales $8 \mathrm{~F}$ y $1492 \mathrm{~F}$. Las secuencias parciales obtenidas fueron comparadas con las disponibles en la base de datos del Gen Bank utilizando el servidor BLASTN (http://www.ncbi.nlm.nih.gov/BLAST/). El análisis de los resultados mostró una coincidencia completa con las secuencias de $K$. rosea mostradas en dicha base. La determinación de la CIM se realizó utilizando tiras de Etest (AB-Biodisk) en agar Mueller Hinton, con una incubación a $35^{\circ} \mathrm{C}$ en aerobiosis durante 16 a 20 horas. Dado que no existen puntos de corte establecidos para este género, para la interpretación se utilizaron los puntos de corte para Staphylococcus sp. según Clinical Laboratory and Standards Institute (CLSI) y el protocolo del fabricante. Los valores de la CIM fueron: vancomicina $1,0 \mu \mathrm{g} / \mathrm{ml}$, daptomicina 0,125 $\mu \mathrm{g} / \mathrm{ml}$, ciprofloxacina $0,5 \mu \mathrm{g} / \mathrm{ml}$, amikacina $1 \mu \mathrm{g} / \mathrm{ml}$, linezolid $0,5 \mu \mathrm{g} / \mathrm{ml}$ y rifampicina $0,004 \mu \mathrm{g} / \mathrm{ml}$. Según el criterio adoptado, el aislado de $K$. rosea fue susceptible a todos los antimicrobianos citados.

Se realizó ecocardiograma doppler bidimensional que no mostró compromiso del endocardio valvular ni mural. Se procedió a retirar el catéter venoso y se indicó tratamiento con vancomicina por 21 días con posterior negativización de los hemocultivos y mejoría clínica y radiológica.

\section{Discusión}

El género Kocuria, llamado así en honor al microbiólogo esloveno Miroslav Kocur, pertenece a la familia Micrococcaceae que incluye varias especies, entre ellas, $K$. rosea, $K$. kristinae, $K$. varians, $K$. palustres, $K$. rhizophila, $K$. aegyptia ${ }^{5}$. Las tres primeras han sido relacionadas con enfermedad en pacientes inmunocomprometidos.

Kocuria rosea fue aislada por primera vez en 1886 por Flügge; se la denominó Micrococcus rosea hasta 1995, año en que recibe su nombre actual ${ }^{6}$. Forma parte de la microbiota normal de la piel, boca y orofaringe de los seres humanos y otros mamíferos por lo que se la considera como una bacteria saprófita. Sin embargo, ante determinadas circunstancias puede actuar como un microorganismo oportunista en sujetos inmunocomprometidos ${ }^{3,4}$.

Lai y cols., analizaron las características clínicas de cinco pacientes con hemocultivos positivos para Kocuria spp. Entre los años 2006 y $2009^{5}$; uno de estos casos fue considerado como una probable contaminación, otro tuvo diagnóstico de endocarditis infecciosa y los tres restantes presentaron bacteriemias asociadas a catéteres venosos centrales (CVC) como puerta de entrada de la infección. Todos los pacientes de esta pequeña serie tenían co-morbilidades subyacentes: cáncer gástrico con nutrición parenteral, dos casos de intestino corto alimentados en forma parenteral y el restante tenía diagnóstico de cáncer de pulmón. En los cuatro pacientes, el CVC debió ser removido con una buena respuesta clínica al tratamiento y sin mortalidad asociada a esta complicación. En todos los pacientes de esta serie, la especie identificada fue Kocuria kristinae y todos eran sujetos inmunocomprometidos. En otro caso publicado previamente ${ }^{4}$, el diagnóstico fue de bacteriemia persistente por $K$. kristinae y la infección no se controló hasta la remoción del CVC. Los casos relatados sugieren que $K$. kristinae es la especie identificada con mayor frecuencia y que la remoción del CVC es una condición necesaria para el control del proceso infeccioso.

Kocuria rosea también ha sido relacionada con episodios de bacteriemia persistente asociada a CVC como en el paciente que describimos. Además del tratamiento antimicrobiano también en el caso que se describe se retiró el catéter vascular. La asociación de la bacteriemia con el CVC, si bien es especulativa ya que los hemocultivos se obtuvieron por punción periférica, resulta útil para alertar sobre la necesidad de su retiro para alcanzar la mejoría del paciente. Sin duda se requiere de un mayor número de casos clínicos bien documentados para concluir sobre la necesidad de retirar los dispositivos intravasculares como parte del tratamiento.

El escaso número de casos publicados evidencia que Kocuria spp. es un patógeno infrecuente en pacientes inmunocomprometidos. En este aspecto, el análisis de la literatura médica señala que parece necesario un factor de inmunocompromiso subyacente además de la presencia de un dispositivo intravascular para desarrollar una infección por microorganismos poco patógenos, como en el caso que se describe. En este contexto clínico debe evaluarse cuidadosamente el valor del hallazgo de este tipo de microorganismos en muestras de hemocultivos. El uso de métodos moleculares es imperativo para el diagnóstico de patógenos inusuales como el descrito ${ }^{5}$.

En relación con el caso que presentamos y, dado que no se comprobó otro foco infeccioso evidente que justificara la descompensación clínica, es posible que el CVC se colonizara con $K$. rosea y desde allí se produjeran episodios de bacteriemia responsables, al menos en parte, del shock séptico del paciente.

Al igual que otras cocáceas grampositivas, $K$. rosea es susceptible a penicilina, vancomicina y quinolonas ${ }^{2,3}$.

En nuestro paciente, la bacteria identificada resultó susceptible a vancomicina, amikacina y ciprofloxacina. Se indicó la primera de estas opciones terapéuticas, con buena respuesta clínica. En la búsqueda bibliográfica efectuada no se hallaron publicaciones de infecciones por este agente en esta población de pacientes.

En conclusión, debido a que se trata de una bacteria cuyo aislamiento e identificación son infrecuentes, consideramos necesario incluir a $K$. rosea como potencial patógeno causante de bacteriemia en pacientes inmunocomprometidos. En este escenario, una demora en el inicio de tratamiento y el desconocimiento del potencial patógeno de $K$. rosea pueden ensombrecer el pronóstico inmediato de estos pacientes.

\section{Referencias bibliográficas}

1.- Stackebrandt E, Koch C, Gvozdiak O, Schumann P. Taxonomic dissection of the genus Micrococcus: Kocuria gen. nov., Nesterenkonia gen. nov., Kytococcus gen. nov., Dermacoccus gen. nov., and Micrococcus Cohn 1872 gen. emend. Int J Syst Bacterial 1995; 45: 682-92.

2. Salas-Segura D. Reporte de un caso de infección por Kocuria rosea. Acta Méd Costarric 2007; 49:170-1.

3.- Altuntas F, Yildiz O, Eser B, Gündogan K, Sumerkan B, Çetin M. Catheter-related bacteremia due to Kocuria rosea in a patient undergoing peripheral blood stem cell transplantation. BMC Infect Dis 2004; 4: 62-4.

4.- Basaglia G, Carreto E, Barbarini D, Moras L, Scalone S, Marone P, et al. Catheter-related bacteremia due to Kocuria kristinae in a patient with ovarian cancer. J Clin Microbiol 2002; 40: 311-3.

5.- Lai CC, Wang JY, Lin SH, Tan CK, Wang CY, Liao CH, et al. Catheterrelated bacteremia and infective endocarditis caused by Kocuria species. Clin Microbiol Infect 2011; 17: 190-2.

6.- Schumann P, Spröer C, Burghardt J, Kovacs G, Stackebrandt E. Reclassification of the species Kocuria erythromyxa (Brooks and Murray 1981) as Kocuria rosea (Flügge 1886). Int J Syst Bacterial 1999; 49: 393. 